\title{
Unir Gamificación y Experiencia de Usuario para mejorar la experiencia docente
}

\section{Gamification and User eXperience for make the learning experience better}

\author{
Emiliano Labrador Ruiz de la Hermosa \\ Eva Villegas Portero \\ La Salle - Universitat Ramon Llull (España)
}

\section{Resumen}

En unos estudios como son las ingenierías TIC se realiza un tipo de asignatura obligatoria y transversal a todos los alumnos. Es una asignatura que trabaja la parte creativa y la parte crítica del alumno, contando que son alumnos con un tipo de perfil de base principalmente tecnológico. Se trata de una asignatura presencial con soporte de una plataforma online que dispone de todos los recursos necesarios para llevarla a cabo. La gamificación forma parte de la asignatura de primer curso de ingeniería, Diseño y usabilidad 1. Su aplicación ha sido diseñada y evaluada para iterar constantemente en la metodología y aplicar cambios continuos que mejoren su diseño y, por lo tanto, que mejoren la aplicación con los alumnos. La gamificación se centra principalmente en respetar el contenido de la asignatura: comunicación, diseño gráfico y usabilidad y experiencia de usuario para tenerlo en cuenta en las mecánicas de juego aplicadas. Tras la gamificación del curso 2013-2014, se realizaron encuestas a los alumnos basadas en técnicas de evaluación de experiencia de usuario para poder obtener datos tanto de la aplicación del contenido académico como de los puntos positivos y negativos de las mecánicas aplicadas. Estas encuestas se contrastaron con los resultados académicos de los alumnos tanto de notas parciales como de notas finales. Este artículo muestra los cambios introducidos en el diseño metodológico, su evaluación y sus posteriores resultados. La pregunta que se pretende responder es: clas mejoras introducidas han hecho que mejoren los resultados?

Palabras clave: juego educativo; usuario; innovación pedagógica; perfil de usuario; motivación.

\begin{abstract}
In studies such as ICT engineering programs, a transversal and compulsory subject is taught to all students. This subject is one, which activates the creativity and the critical capacity of the student, given that the majority of the students come from a more technological background. Gamification forms part of the Design and Usability 1 course. It has been designed and evaluated to be used repeatedly in the methodology and changes have been applied which have improved its design and its application with students. Gamification is based mainly on respecting the course content: communication, graphic design and usability and user experience to be taken into consideration in the mechanisms of applied games.
\end{abstract}


After the gamification in the 2013-14 course, students were surveyed on the user experience evaluation techniques in order to obtain data on both the academic content and the positive and negative points of the mechanisms applied. These surveys were compared to the academic results of the students in the both the semester and final exams. This article shows the changes introduced in the design methodology, its evaluation and the consequent results. The question to be asked is: have the improvements made to the program improved the results?

Keywords: educational game; user; educational innovation; user profile; motivation.

La asignatura Diseño y usabilidad 1 de La Salle-Universitat Ramon Llull es una asignatura de primero de grado, común a las 7 ingenierías TIC que se imparten: grado en ingeniería de telecomunicación, telemática, electrónica, sistemas audiovisuales, organización de las TIC, informática y multimedia. En esta asignatura se imparten conocimientos de tres áreas: Comunicación, Diseño gráfico y Usabilidad y experiencia de usuario. Durante el primer semestre se tratan como disciplinas independientes, de forma que los alumnos adquieran las habilidades básicas de cada una de ellas y en el segundo semestre se realiza la integración de las tres, mediante una práctica grupal que implica un diseño usable y su posterior comunicación a un cliente ficticio, tanto en forma oral como escrita.

La asignatura se imparte desde el curso 09/10 y desde entonces se ha mantenido la metodología: presencial y con recursos adicionales en línea, así como la teoría y las prácticas relacionadas. Si bien a lo largo de los años se ha ido actualizando el contenido, la esencia de la asignatura es la misma, así como los profesores que la imparten y los criterios de evaluación.

La asignatura, cuya naturaleza es creativa, y está más cercana a la percepción humana que a las ciencias exactas, es percibida por muchos alumnos como una asignatura a la que no es necesario dedicar tiempo. Esto constituye un grave error que tiene como resultado una gran tasa de abandono de la convocatoria oficial al comprobar los alumnos que unos días antes de la fecha de entrega de los ejercicios, no es posible asumir las tareas requeridas. Este hecho se ha repetido años tras año, llegando a un pico muy bajo en el curso 12/13. Fue el momento en que se decidió hacer un cambio para revertir esta situación. El claustro de profesores creía firmemente en el contenido de la asignatura, por lo que la decisión fue cambiar la metodología.

Los alumnos de esta clase tienen, en su gran mayoría, 18 años. Son, pues, la generación llamada Generación Y o millennials. Es una de las primeras generaciones nativa digital, mientras que los formadores son en su mayoría de una generación anterior que no ha entendido los cambios que la informática ha introducido en sus vidas (Fructuoso, 2015). Los millennials basan su forma de vida en la misma forma en que lo han aprendido del entorno informatizado en el que han crecido. Así pues, la libertad de decisión, la inmediatez, la toma rápida de decisiones, la personalización, el gusto por trabajar para aprender o realizarse más que por dinero... son algunas de esas decisiones vitales que conforman su día a día. Y en esto han influido no poco 
los videojuegos con los que se han educado desde su nacimiento (Howe, Strauss y Matson, 2000).

Los millennials son una generación acostumbrada a jugar y a dar una respuesta automática cuando se enfrentan a una mecánica de juego, independientemente del entorno en que la encuentren. Esta conversión de cualquier proceso (aprendizaje, mejora de la salud, fidelización de clientes,...) en un sistema provisto de mecánicas de juego (Kapp, 2012), ha demostrado durante los últimos años que funciona. Y no sólo con la generación Y. La gamificación ha existido desde siempre. Los cupones del supermercado, el periodo limitado de rebajas,... son estrategias de gamificación que no son nuevas, aunque la etiqueta con las que se nombran sí lo sea. La proliferación de juegos y estudios sobre el fenómeno ha dado como resultado una sistematización de los conceptos usados y una nomenclatura que ha sido rápidamente aprovechada por los profesionales para usarlos en ámbitos no lúdicos, dando unas consecuencias espectaculares, tanto en los resultados como en la aceptación popular. En este sentido, hay estudios que demuestran los beneficios de jugar a videojuegos para el cerebro (Kühn, Gleich, Lorenz, Lindenberger y Gallinat, 2014), creando plasticidad y desarrollando habilidades como toma de decisiones rápidas, mejoras en la memoria, la concentración o la rapidez visual entre otras. Además, los videojuegos se juegan por placer. Contienen mecánicas que activan la motivación intrínseca (Valderrama, 2010), la que se realiza por propio convencimiento de que el acto será placentero o útil (Brophy, 2004; Harlen, 2003). De este modo, los jugadores pueden estar jugando durante horas sin caer en el desánimo o el aburrimiento. Para los autores era fundamental promover la motivación intrínseca (Mekler, Brühlmann, Tuch y Opwis, 2015) y que los alumnos entendieran la importancia del aprendizaje de sus contenidos. Si bien sí que se instauró una mecánica que ayudara como motivador extrínseco, los puntos, todas las demás, y en las que se hace más énfasis, están enfocadas a la motivación intrínseca. Estos motivos llevaron a decidir realizar un cambio metodológico que fuera en esta línea. La gamificación ya había sido probada en el aula, tanto a nivel metodológico, como en el caso del framework ClassCraft (Young, 2013), donde se plantea convertir la clase en un complejo juego de rol, o a nivel de prácticas e investigación (Smith-Robbins, 2011; Lee y Hamme, 2011), donde se demuestran los beneficios de usar estas técnicas en el aprendizaje.

Así pues, mientras los juegos aplican las mecánicas, la historia, la tecnología y estética (Schell, 2008) de forma holística, en gamificación se emplean de forma seccionada, aplicando los elementos necesarios en el momento necesario. Este hecho es muy útil, ya que cada mecánica se puede asociar a una carencia del curso, atacando cada uno de los objetivos marcados de forma individual, y no teniendo que crear un videojuego complejo, que por recursos, tanto económicos como de tiempo, no hubiera sido posible. 


\section{MÉTODO}

Los profesores de la asignatura, autores de este artículo, son también miembros del grupo de investigación de tecnologías media de La Salle-URL ${ }^{1}$. Eva Villegas coordina la sección de Experiencia de Usuario (UX) (Krug, 2006) donde se realizan tareas tanto comerciales como de investigación en metodologías de captación y análisis de datos de usuarios. En este contexto, se han desarrollado metodologías de indagación propias como la entrevista Bipolar Laddering (BLA) (Pifarré, Sorribas y Villegas, 2009; Sanders, 2005). El BLA es un tipo de entrevista socrática (Guidano, 1989) donde los usuarios tienen que valorar tres aspectos positivos y tres negativos del sistema que hayan usado, explicar el porqué de esa valoración y proponer una solución. Los usuarios no son coaccionados en ningún modo, es decir, no se les hace ninguna pregunta sobre aspectos concretos. Son los propios usuarios los que libremente mencionan un aspecto u otro. De este modo, se obtienen una serie de datos cuantitativos (índice de mención, valoración de estos) y cualitativos, a través de los literales que se recogen.

En este contexto, se decidió trabajar en una metodología (Gauntlett, 2007) que uniera ambas disciplinas (Sanders, 2005). La gamificación cuenta con un nutrido número de profesionales, pero no de investigadores, por lo que su base científica es a veces discutida (Lee y Hamme, 2011). Poniendo el foco en esta necesidad, se aplicaron técnicas de extracción y análisis de datos propios de UX (Jorgensen, 1989) durante el proceso de creación (Jensen, 2004) de la gamificación de la asignatura, surgiendo así la metodología Fun Experience Design (FED) (Labrador y Villegas, 2014). La metodología FED sirve para crear sistemas gamificados, teniendo en cuenta las necesidades de los usuarios desde el primer momento. Como se puede ver en la figura 1, se divide en 4 fases. La primera es una fase de exploración (Neimeyer, 1995), donde se obtienen los datos demográficos de los usuarios, así como los datos de interés para el diseño del sistema: qué es lo que los usuarios que actualmente están usando el sistema, en este caso, los alumnos de la asignatura a gamificar, piensan sobre la experiencia. En este caso, se aplicaron la entrevista BLA y un cuestionario de valoración emocional, donde a los alumnos se les muestran una serie de parejas de emociones opuesta y escogen en qué porcentaje están más cerca una de otra. 
Figura 1. Representación gráfica del sistema Fun Experience Design (FED)

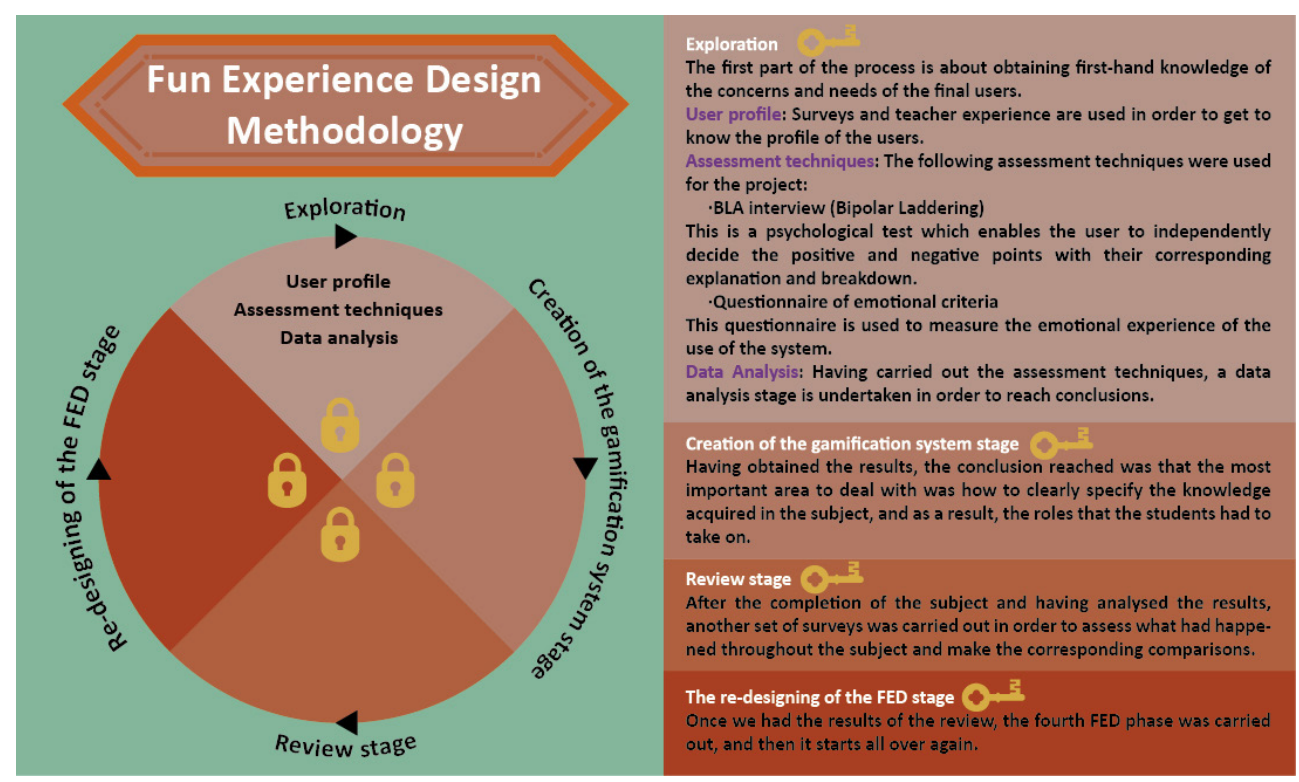

Tras el análisis de estos datos, se pasa a la fase de creación de las mecánicas que se usarán en clase. Se crearon diferentes mecánicas para satisfacer diferentes objetivos. Se puede leer una lista exhaustiva en las actas del congreso ICEILT14 (Labrador y Villegas, 2014). Una de las más relevantes, y centro de este artículo, fue la creación de modos que explicitaran el tipo de conocimiento que el alumno estaba adquiriendo. $\mathrm{Al}$ equipo de docentes nos parecía fundamental que el alumnado tomara conciencia de la utilidad de la asignatura y de los diferentes roles de la industria que acabarían adquiriendo al finalizar el curso.

La implementación de la Gamificación se hizo empleando elementos básicos que está comprobado que funcionan. Para una primera versión se pensó en el uso de medallas, puntos y leaderboards asociadas a retos (Seaborn y Fels, 2015), ya que han demostrado su eficacia en el aumento de la motivación de usuarios tanto en entornos no educativos (Hamari, 2015; Deterding, Sicart, Nacke, O’Hara y Dixon, 2011) como en entornos educativos (Hamari y otros, 2015). Si bien el uso de medallas da visibilidad a los logros personales de una forma abstracta, los leaderboards son un elemento más controvertido ya que se produce una comparación directa entre alumnos que no siempre puede tener los efectos deseados. Si bien en otros entornos pueden ser útiles (Landers y Bauer, 2015), no siempre es así (Landers y Landers, 2015) y los autores quisieron emplearlo de forma más sutil, dada la experiencia previa en otras asignaturas. De este modo, aunque se implementó el leaderboard, no se mostró a los alumnos de forma ordenada por los puntos logrados, sino por orden 
alfabético, de forma que la comparación era posible, pero no era directa, estando las diferentes posiciones mezcladas.

Así, se crearon las siguientes mecánicas:

\section{Medallas}

Las medallas tienen dos funciones. Por un lado, explicitar el nivel de profundidad de cada trabajo del alumno, de forma que, al margen de la nota numérica, tengan un feedback que les haga entender de una forma más clara en qué han fallado y qué es mejorable. Por otro, tener una visibilidad del progreso de los conocimientos. Los niveles están basados en la acumulación de medallas.

Las medallas (tablas 1, 2 y 3) se consiguen dependiendo de la nota sacada en cada ejercicio o práctica, divididas en tres franjas: excelente, aceptable y no aceptable. Algunos ejercicios o prácticas hacen conseguir medallas de dos o más conceptos.

Tabla 1. Medallas de la categoría de Diseño

\begin{tabular}{|l|l|}
\hline & $\begin{array}{l}\text { Creativo: Todas tus ideas son originales y parten de una interpretación } \\
\text { personal de tus conocimientos y tus investigaciones. }\end{array}$ \\
\hline $\begin{array}{l}\text { Reciclado: Las ideas que realizas están basadas en otras ya preexistentes } \\
\text { y aunque les das un cierto nivel de personalización, se nota la fuente } \\
\text { original. }\end{array}$ \\
\hline $\begin{array}{l}\text { Conformista: No se precia un esfuerzo por realizar ideas propias. Usas } \\
\text { fuentes preexistentes sin ningún intento apreciable de personalización. }\end{array}$ \\
\hline
\end{tabular}

Tabla 2. Medallas de la categoría de Usabilidad

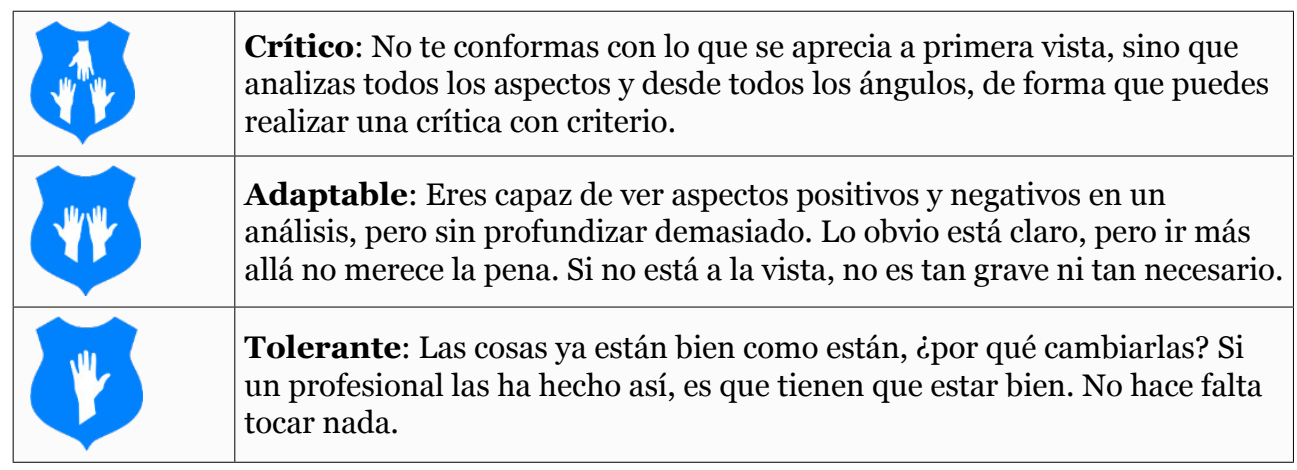


Tabla 3. Medallas de la categoría de Comunicación

\begin{tabular}{|l|l|}
\hline & $\begin{array}{l}\text { Comunicador: Eres capaz de comunicar ideas de una forma amena } \\
\text { y agradable. Puedes transmitir información relevante a la vez que } \\
\text { entretienes. }\end{array}$ \\
\hline $\begin{array}{l}\text { Sociable: La información que transmites es entendible y la audiencia la } \\
\text { recibe con agrado. Sería conveniente entregarse más al público, aunque el } \\
\text { mensaje ha quedado claro. }\end{array}$ \\
\hline $\begin{array}{l}\text { Huraño: El mensaje no ha llegado a la audiencia. Quizá lo que tienes que } \\
\text { transmitir es interesante, pero no se puede percibir como tal. }\end{array}$ \\
\hline
\end{tabular}

\section{Niveles}

Cada una de las partes de la asignatura, tanto diseño como usabilidad, tiene unos objetivos particulares que se han dividido en 3 niveles de logro, desde el ideal que se pretende conseguir hasta el más deficiente.

Los objetivos de la asignatura son que el alumno alcance el nivel máximo de creatividad y capacidad de crítica, así como unas habilidades óptimas de comunicación, oral y escrita. No obstante, como se vio en el apartado anterior, se establecen diferentes niveles de logro de objetivos dependiendo de las diferentes combinaciones que se pueden realizar. Las combinaciones y sus denominaciones quedan organizadas en la tabla 4, teniendo en cuenta sólo las capacidades referidas a Diseño y Usabilidad y no la de comunicación para no tener un listado de niveles demasiado complejo:

Tabla 4. Niveles a partir de la combinación de usabilidad y diseño

\begin{tabular}{|l|l|l|l|}
\hline & $\begin{array}{l}\text { Creativo } \\
\text { (4-5 CRE) }\end{array}$ & $\begin{array}{l}\text { Reciclado } \\
\text { (3-2 CRE) }\end{array}$ & $\begin{array}{l}\text { Conformista } \\
\text { (1-o CRE) }\end{array}$ \\
\hline $\begin{array}{l}\text { Crítico } \\
(4-5 \text { CRI })\end{array}$ & EDEN &
\end{tabular}




\begin{tabular}{|l|lll}
\hline $\begin{array}{l}\text { Adaptable } \\
\text { (3-2 CRI) }\end{array}$ \\
$\begin{array}{l}\text { Tolerante } \\
\text { (1-O CRI) }\end{array}$
\end{tabular}

\section{Clanes}

La asignatura de Diseño y usabilidad 1 forma a los alumnos en tres aspectos (ver tabla 5):

Tabla 5. Los tres perfiles en los que se forma al alumno

Técnico: Te gusta realizar el aspecto tecnológico, ya sea físico,
telemático o de programación de un proyecto.
Artista: Te gusta realizar el aspecto visual, narrativo y/o estético de un
proyecto.

La asignatura parte de conocimientos básicos, por lo que se presupone que el alumno no tiene nociones en ninguno de estos aspectos. Así, se comienza el curso sin un tipo de rol específico y a medida que se vayan adquiriendo conocimientos se producirá un cambio en los roles. Aunque lo ideal es que se acabe el curso asumiendo el rol más completo, el que implica obtener conocimientos en los tres ámbitos, el no 
alcanzar alguno de ellos, teniendo en cuenta los diferentes perfiles de entrada y las especialidades escogidas, no es muy relevante.

De este modo, se establecen los diferentes clanes (tabla 6) que dan las combinaciones, en este caso sí, de las 3 habilidades.

Tabla 6. Descripción de clanes y sus iconos

\begin{tabular}{|c|c|c|c|}
\hline & & \\
\hline $\begin{array}{c}\text { Eécnico + Artista } \\
+ \text { Comunicador }\end{array}$ & $\begin{array}{c}\text { Técnico }+ \\
\text { Artista }\end{array}$ & $\begin{array}{c}\text { Técnico + } \\
\text { Comunicador }\end{array}$ & $\begin{array}{c}\text { Artista }+ \\
\text { Comunicador }\end{array}$ \\
\hline \hline & & & \\
\hline Minotauro & Fénix & Hidra & Humano \\
\hline Técnico & Artista & Comunicador & Nada \\
\hline
\end{tabular}

\section{RESULTADOS}

Estas mecánicas, unidas a las otras que se usaron durante el curso, dieron muy buenos resultados a nivel de notas obtenidas por los alumnos. Para más información sobre este punto se puede consultar el artículo de los autores en el congreso ICEILT14 (Labrador y Villegas, 2014).

Este artículo se centra en la adquisición de perfiles por parte de los alumnos.

Desde la creación de la asignatura, formar en los tres perfiles definidos al comienzo del artículo fue el objetivo principal de la asignatura. Sin embargo, nunca se realizó un sistema que pudiera medir con exactitud el porcentaje de adquisición de estos perfiles. Uno de los objetivos de la gamificación fue, pues, explicitar de forma clara qué tipo de conocimiento se estaba adquiriendo. Los resultados del curso 13/14 fueron los que se muestran en la tabla 7. 
Tabla 7. Resultados por niveles en el curso 13/14

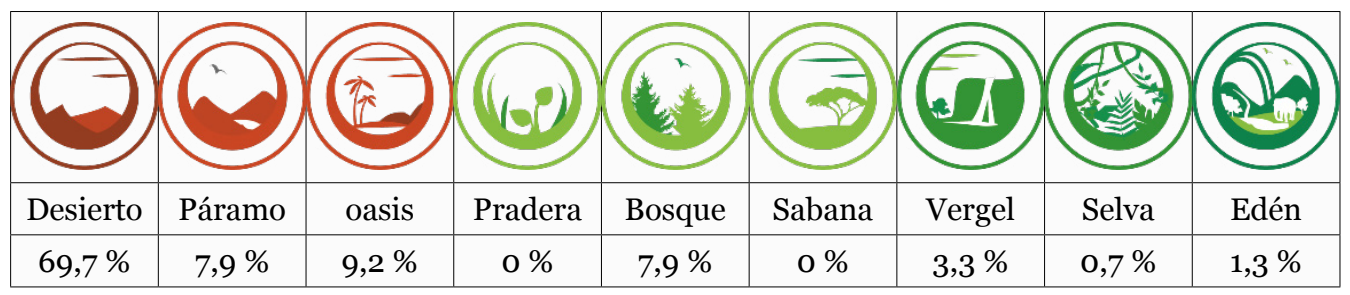

Al finalizar el curso, y a pesar de haber subido considerablemente la nota media del curso, un 69.7\% de la clase no había alcanzado ningún nivel. Es decir, no había conseguido ninguna combinación de medallas con valor significativo. El resto de la clase, poco más de la cuarta parte, habían tenido una adquisición de perfiles muy heterogénea, reflejado en que se han conseguido diferentes tipos de medallas, aunque todas en un porcentaje bastante bajo.

Así pues, los clanes a los que los alumnos se pudieron unir, es decir, los roles que adquirieron de forma satisfactoria, son los que se muestran en la tabla 8 .

Tabla 8. Resultados por perfiles en el curso 13/14

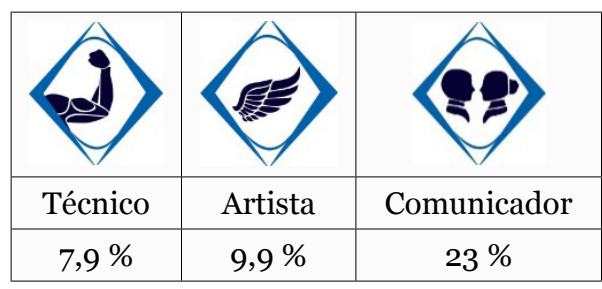

Los porcentajes de adquisición de un rol concreto son muy bajos, por debajo del 10\% en el caso de técnico y artista, y aunque sube considerablemente en el caso de la comunicación, hasta un $23 \%$, sigue siendo un valor poco satisfactorio. Hay que tener en cuenta, además, que un alumno puede tener más de un perfil. Los valores de la combinación de los perfiles, se refleja en la tabla de los perfiles, tabla 9: 
Tabla 9. Resultados por clanes en el curso 13/14

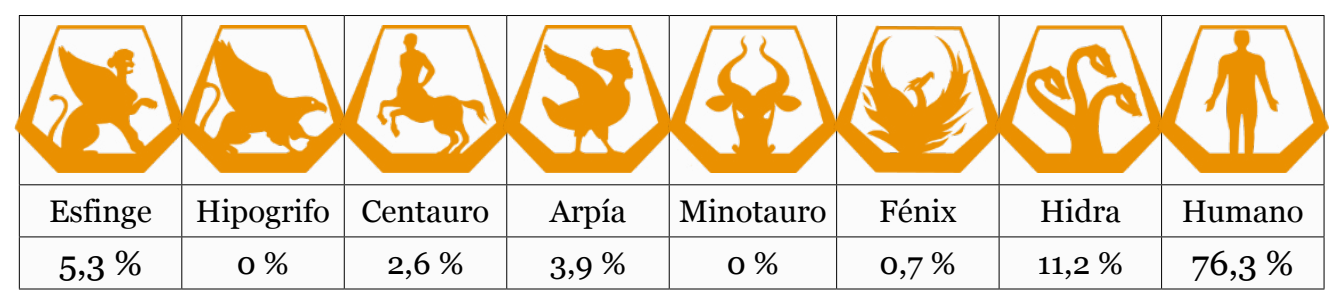

La tabla 9 muestra como más de tres cuartos de la clase no ha adquirido ningún perfil con suficiente calidad. Apenas algo más de un 10\% ha adquirido el perfil de comunicador, y un número mínimo o nulo los perfiles únicos de artista y técnico. Los valores de la combinación de dos de los perfiles también son muy bajos. Sin embargo, la combinación de los tres perfiles (esfinge) ha sido adquirida por un número mayor de alumnos que la combinación de dos.

Una vez comprobados los datos y analizados los resultados, se verificó que, aunque las notas finales eran satisfactorias, la adquisición de perfiles concretos no se estaba realizando. Es decir, los alumnos tenían una nota media de todos los ejercicios que les permite pasar la asignatura, pero su conocimiento era de elementos puntuales, sin coherencia entre ellos.

El índice de alumnos que acabaron no presentándose a la convocatoria ordinaria fue del $14.4 \%$, un porcentaje bastante elevado teniendo en cuenta que uno de los objetivos de la gamificación era reducir en gran medida el porcentaje de abandono. Sí que se logró reducir el porcentaje respecto a los años en los que no se había aplicado gamificación, pero no en la medida esperada.

Para cambiar esto, se tomaron dos medidas.

Por un lado, se decidió ajustar la franja de notas numéricas que había que superar para conseguir cada medalla.

Durante el curso 13/14 la valoración se muestra en la tabla 10.

Tabla 10. Valoración de las medallas respecto a las notas en el curso 13/14

\begin{tabular}{|l|l|}
\hline Creativo & $9-10$ \\
\hline Reciclado & $6-8$ \\
\hline Conformista & $1-5$ \\
\hline Crítico & $9-10$ \\
\hline adaptable & $6-8$ \\
\hline Tolerante & $1-5$ \\
\hline comunicador & $9-10$ \\
\hline sociable & $6-8$ \\
\hline huraño & $1-5$ \\
\hline
\end{tabular}


Y para el curso 14/15, viendo que los resultados de las notas no habían sido tan malos, se ajustó para valorar mejor los ejercicios presentados, quedando la tabla 11:

Tabla 11. Valoración de las medallas respecto a las notas en el curso 14/15

\begin{tabular}{|l|l|}
\hline Creativo & $8.5-10$ \\
\hline Reciclado & $6-8.4$ \\
\hline Conformista & $1-5 \cdot 9$ \\
\hline Crítico & $8.5-10$ \\
\hline adaptable & $6-8.4$ \\
\hline Tolerante & $1-5.9$ \\
\hline comunicador & $8.5-10$ \\
\hline sociable & $6-8.4$ \\
\hline huraño & $1-5.9$ \\
\hline
\end{tabular}

Además, se decidió cambiar los ejercicios de evaluación continua para que los mismos alumnos pudieran participar en una experiencia más lúdica. Los diferentes ejercicios que se venían aplicando desde el comienzo de la asignatura, ejercicios donde se ponían en práctica la adquisición de conocimientos de la teoría y de las herramientas, se sustituyeron por la elaboración por parte de los alumnos de una serie de pasatiempos (crucigramas, sopas de letras, quiere ser millonario,...) donde las preguntas a resolver tenían que hacer referencia a la teoría de la asignatura. De este modo, se les obligaba a tener que estudiar la teoría antes del examen y a poner en práctica herramientas de dibujo para realizar los pasatiempos.

Tras aplicar estos cambios durante el curso 14/15, los resultados obtenidos con respecto a las notas fueron similares, con alguna ligera mejora respecto al curso anterior (Labrador y Villegas, 2014). En cuanto a la adquisición de perfiles, quedó como se muestra en la tabla 12:

Tabla 12. Resultados por niveles en el curso 14/15

\begin{tabular}{|c|c|c|c|c|c|c|c|c|}
\hline & & & \\
\hline
\end{tabular}


Los alumnos en el nivel Desierto han disminuido y han aumentado prácticamente todos los otros, es decir, más alumnos han acabado adquiriendo un perfil concreto dentro de la asignatura. Aun así, los porcentajes aún son muy bajos.

En cuanto a los clanes finales, el último recuento se indica en la tabla 13:

Tabla 13. Resultados por perfiles en el curso 14/15

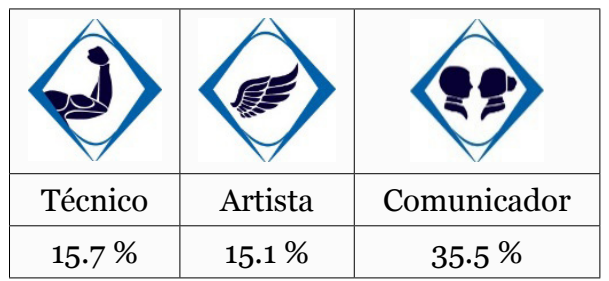

También se puede apreciar cómo los tres valores han subido de forma significativa. Finalmente, la combinación de perfiles se ve en la tabla 14:

Tabla 14. Resultados por clanes en el curso 14/15

\begin{tabular}{|c|c|c|c|c|c|c|c|}
\hline Esfinge & Hipogrifo & Centauro & Arpía & Minotauro & Fénix & Hidra & Humano \\
\hline $8.7 \%$ & $0.6 \%$ & $5.8 \%$ & $4.1 \%$ & $0.6 \%$ & $1.7 \%$ & $16.9 \%$ & $61.6 \%$ \\
\hline
\end{tabular}

Donde se puede apreciar en igual modo que el porcentaje de humanos, es decir, alumnos que no han adquirido ningún perfil concreto ha disminuido ligeramente y todos los demás valores han aumentado.

Por otro lado, el número de alumnos que no se presentaron a la convocatoria ordinaria sufrió un ligero descenso, aunque no demasiado significativo. Fue el 12.2 $\%$ de los alumnos.

\section{DISCUSIÓN}

Sin duda alguna, la implementación de la metodología basada en la gamificación surtió el efecto deseado en cuanto al aumento del rendimiento de los alumnos (Labrador y Villegas, 2014). 
En cuando a la adquisición de niveles, en la tabla 15 podemos ver los incrementos relativos de cada uno de ellos.

Tabla 15. Comparativa de niveles en los dos años analizados

\begin{tabular}{cccccccccc}
\hline & Desierto & Páramo & oasis & Pradera & Bosque & Sabana & Vergel & Selva & Edén \\
\hline $13 / 14$ & $69,7 \%$ & $7,9 \%$ & $9,2 \%$ & $0 \%$ & $7,9 \%$ & $0 \%$ & $3,3 \%$ & $0,7 \%$ & $1,3 \%$ \\
$14 / 15$ & $61.6 \%$ & $8.7 \%$ & $5.2 \%$ & $0 \%$ & $15.7 \%$ & $0 \%$ & $3,5 \%$ & $3.5 \%$ & $1,7 \%$ \\
$\begin{array}{c}\text { Incremento } \\
\text { relativo }\end{array}$ & $-11,6 \%$ & $10,1 \%$ & $-43,5 \%$ & $0 \%$ & $98,7 \%$ & $0 \%$ & $6,1 \%$ & $400 \%$ & $30,8 \%$ \\
\hline
\end{tabular}

Se puede observar que, dejando aparte el desierto, el nivel del que partían todos los alumnos -a excepción de uno, el oasis- todos los niveles se han incrementado, algunos de ellos significativamente. Esto significaría, por un lado, que el ajuste de asignación de medallas por las notas numéricas ha hecho que más alumnos opten a mejores medallas, incrementándose los niveles, clanes y perfiles. Por otro lado, el cambio en los ejercicios de evaluación continua también ha podido ser más motivador para los alumnos, ayudando a que se realizaran mejores prácticas. El ajuste de la asignación no fue tan exagerado como para que por sí solo haya justificado un cambio tan grande.

También es curioso observar que los niveles Pradera (Conformista+Crítico) y Sabana (Tolerante+Creativo) no han sido obtenidos por ningún alumno en ninguna de las dos ediciones, siendo Bosque una posición intermedia (Reciclado+Adaptable), que ha sufrido uno de los mayores incrementos.

En la tabla 16 podemos comprobar cómo los incrementos relativos de los roles también han sufrido una variación muy positiva. Los alumnos han asumido cada uno de los tres roles en mayor cantidad que el año anterior. Las cifras son similares en comunicador y artista, pero casi se dobla en el caso del rol de técnico. Las cifras comienzan a ser más aceptables, aunque aún son ampliamente mejorables.

Tabla 16. Comparativa de perfiles en los dos años analizados

\begin{tabular}{cccc}
\hline & Técnico & Artista & Comunicador \\
\hline $13 / 14$ & $7,9 \%$ & $9,9 \%$ & $23 \%$ \\
$14 / 15$ & $15.7 \%$ & $15.1 \%$ & $35.5 \%$ \\
Incremento relativo & $98.7 \%$ & $58.6 \%$ & $54.3 \%$ \\
\hline
\end{tabular}


La combinación de roles, los clanes, también se ha visto afectada. En la tabla 17 podemos ver cómo todas las categorías han aumentado, algunas de forma significativa, como el fénix o el centauro con un aumento mucho mayor del $100 \%$. Algunas categorías que no tuvieron representación en el curso anterior, aunque con un porcentaje mínimo, aparecen, e incluso la categoría esfinge, la que engloba los tres perfiles, aumenta de forma significativa. La única categoría que disminuye es la de humano, con la que se empieza el curso y en la que no debería quedar nadie a final de curso. Incluso bajando esta, aún tiene un porcentaje muy elevado.

Tabla 17. Comparativa de clanes en los dos años analizados

\begin{tabular}{ccccccccc}
\hline & Esfinge & Hipogrifo & Centauro & Arpía & Minotauro & Fénix & Hidra & Humano \\
\hline $13 / 14$ & $5,3 \%$ & $0 \%$ & $2,6 \%$ & $3,9 \%$ & $0 \%$ & $0,7 \%$ & $11,2 \%$ & $76,3 \%$ \\
$14 / 15$ & $8.7 \%$ & $0.6 \%$ & $5.8 \%$ & $4.1 \%$ & $0.6 \%$ & $1.7 \%$ & $16.9 \%$ & $61.6 \%$ \\
$\begin{array}{c}\text { Incremento } \\
\text { relativo }\end{array}$ & $64.2 \%$ & - & $123.1 \%$ & $5.1 \%$ & - & $142.9 \%$ & $50.9 \%$ & $-19.3 \%$ \\
\hline
\end{tabular}

Finalmente, la tabla 18 muestra los alumnos no presentados.

Tabla 18. Comparativa de alumnos No Presentados en los dos años analizados

\begin{tabular}{cc}
\hline & NP \\
\hline $13 / 14$ & 14,5 \\
$14 / 15$ & 12,2 \\
Incremento relativo & $-15,9$ \\
\hline
\end{tabular}

La tendencia, una vez más, es a la baja, pero el porcentaje sigue siendo demasiado elevado. El porcentaje de alumnos que abandonan la asignatura es aún alto.

Analizados todos estos datos, se puede concluir que la implementación de la metodología basada en la gamificación ha funcionado bien con los estudiantes que vienen predispuestos a estudiar, como se demuestra en el aumento del perfil Esfinge, pero no funciona con aquellos que no vienen predispuestos a estudiar, como refleja el número de NP, que se mantiene casi igual.

La gamificación ha ayudado a que los alumnos aprendan de forma más efectiva los roles de la industria y además se les ha explicitado, de forma que ellos son conscientes de sus fortalezas y debilidades, mucho más allá de una simple nota numérica final, que no da idea de la adquisición real de conocimiento. 


\section{LÍNEAS DE FUTURO}

Como líneas de futuro, se plantean dos ideas:

Cambio de nuevo de las prácticas de evaluación continua. Si bien tanto la asunción de perfiles como las notas numéricas han sufrido un aumento, los alumnos no han valorado positivamente el realizar pasatiempos con la teoría, ya que lo ven muy alejado de su actividad profesional. Se plantea para el siguiente curso actividades que pongan en práctica igualmente tanto el uso de herramientas como el estudio de la teoría en clase, pero que tengan una forma que se perciba como más útil para los estudiantes.

Por otro lado, se marca como objetivo prioritario no tanto la motivación de todo el alumnado, que con las mecánicas actuales ya funcionan de modo adecuado, sino de aquellos estudiantes que no van a clase motivados y no suelen participar de las dinámicas diarias, ya que son los que finalmente acaban abandonando la asignatura. Para todos los alumnos, pero haciendo énfasis en este último segmento, se plantea una nueva mecánica que es el uso de cartas físicas donde se premie el esfuerzo diario en clase, de forma que vean una recompensa inmediata a su esfuerzo y se animen a continuar con el estudio fuera del aula.

\section{NOTAS}

1. http://lasallerd.salleurl.edu/

\section{REFERENCIAS BIBLIOGRÁFICAS}

Brophy, J. (2004). Motivating students to learn. Mahwah, New Jersey: Lawrence Erlbaum Associates.

Deterding, S., Sicart, M., Nacke, L., O’Hara, K., y Dixon, D. (2011). Gamification: Using Game Design Elements in NonGaming Contexts. Workshop at CHI. Vancouver, BC, Canada.

Fructuoso, I. N. (2015). How Millennials are changing the way we learn: the state of the art or ICT integration in education. RIED, Revista Iberoamericada de Educación a Distancia, 18(1), 45-65.

GameWise. (2014). GameWise. Recuperado de http://game-wise.eu/

Gauntlett, D. (2007). Creative Explorations: New Approaches to Identities and Audiences. Routledge.
Guidano, V. (1989). Constructivist psychotherapy: A theoretical framework. In R. A. Neimeyer y M. J. Mahoney (Eds.), Constructivism in Psychotherapy. Cambridge: Cambridge University Press.

Hamari, J. (2015). Do badges increase user activity? A field experiment on effects of gamification. Computers in human behavior.

Hamari, J., Shernoff, D. J., Rowe, E., Coller, B., Asbell-Clarke, J., y Edwards, T. (2015). Challenging games help students learn: An empirical study on engagement, flow and immersion in game-based learning. Computers in Human Behavior.

Harlen, R. D. (2003). Testing and Motivation for Learning. Assessment in Education, 1O(2), 169-207. 
Howe, N., Strauss, W., y Matson, R. (2000). Millennials Rising: The Next Great Generation. New York: Vintage Books.

Jensen, B. G. (2004). The role of the artifact in participatory design research. In Design communication. 3rd Nordcode Seminar\&Workshop. Lyngby-Denmark.

Jorgensen, D. (1989). Participant Observation: A Methodology For Human Studies. Newbury Park, CA: Sage Publications.

Kapp, K. M. (2012). The Gamification of Learning and Instruction: Game-based Methods and Strategies for Training and Education. USA: Pfeiffer.

Krug, S. (2006). No me hagas pensar. Madrid: Pearson educación.

Kühn, S., Gleich, T., Lorenz, R., Lindenberger, U., y Gallinat, J. (2014). Playing Super Mario induces structural brain plasticity: gray matter changes resulting from training with a commercial video. Molecular Psychiatry, 19, 265-271.

Labrador, E., y Villegas, E. (2014). Fun Experience Design Applied to Learning. ICEILT, 14.

Landers, R. N., y Bauer, K. (2015). Gamification of task performance with leaderboards: A goal setting experiment. Computers in Human Behavior.

Landers, R. N., y Landers, A. K. (2015). An Empirical Test of the Theory of Gamified Learning: The Effect of Leaderboards on Time-on-Task and Academic Performance. Simulation and Gaming, 769-785.

Lee, J. J., y Hamme, J. (2011). Gamification in Education: What, How, Why Bother? Recuperado de http://www.academia. edu/570970/Gamification in Education What How Why Bother
Mekler, E. D., Brühlmann, F., Tuch, A. N., y Opwis, K. (2015). Towards understanding the effects of individual gamification elements on intrinsic motivation and performance. Computers in Human Behavior.

Neimeyer, R. A. (1995). Features, foundations and future directions. In R. A. Neimeyer, M. J. Mahoney (Eds.), Constructivism in Psychotherapy. Washington: American Psychological Association.

Pifarré, M., Sorribas, X., y Villegas, E. (2009). BLA (Bipolar Laddering) Applied to YouTube. Performing Postmodern Psychology Paradigms in User Experience Field. In Advances technologies. Kankesu Jayanthakumaran.

Sanders, E. (2005). Information, Inspiration and Co-creation. Proceeding of the 6th International Conference of the European Academy of Design. Bremen, University of the Arts.

Schell, J. (2008). The art of game design. A book of lenses. Amstermam/Boston: Elsevier/ Morgan Kaufmann.

Seaborn, K., y Fels, D. I. (2015). Gamification in theory and action: A survey. International Journal of HumanComputer Studies, 74, 14-31.

Smith-Robbins, S. (2011). This Game Sucks: How to Improve the Gamification of Education. EDUCAUSE Review, 467(1), 58-59.

Valderrama, B. (2010). Motivacion inteligente. Prentice-Hall.

Young, S. (2013). Classcraft. Make learning an adventure. Recuperado de http:/ www.classcraft.com/es/ 


\section{PERFIL ACADÉMICO Y PROFESIONAL DE LOS AUTORES}

Emiliano Labrador. Graduado en Multimedia por la UOCy Máster en Creación e Ingeniería Multimedia por La Salle-URL. Actualmente es director del Máster en Creación Multimedia y Serious Games (MCDEM) en La Salle-URL y forma parte del Grup de Recerca en Tecnologies Mèdia (GTM) (2014-SGR-590). Su principal interés de investigación es la aplicación de mecánicas de juego para mejorar la experiencia del usuario en diversos ámbitos, con especial énfasis en la educación.

E-mail:eruiz@salleurl.edu

Eva Villegas. Graduada en Multimedia por la UPC, Máster en Creación Multimedia y Serious Games en La Salle- URL y Máster en Tecnologías Accesibles para la Sociedad de la Información en la UOC. Actualmente es coordinadora y profesora del Grado en Ingeniería Multimedia y del Master en User eXperience. Forma parte del Grup de Recerca en Tecnologies Mèdia (2014-SGR-590). Su investigación se centra en la creación de nuevas metodologías de evaluación de experiencia de usuario.

E-mail: evillegas@salleurl.edu

\section{DIRECCIÓN DE LOS AUTORES}

La Salle

Universitat Ramon Llull

Quatre Camins, 30, 08022

Barcelona (España)

Fecha de recepción del artículo: 11/12/2015

Fecha de aceptación del artículo: 21/01/2016

\section{Como citar este artículo:}

Labrador, E., y Villegas, E. (2016). Unir Gamificación y Experiencia de Usuario para mejorar la experiencia docente. RIED. Revista Iberoamericana de Educación a Distancia, 19(2), pp. 125-142. doi: http://dx.doi.org/10.5944/ried.19.2.15748 